\title{
Interference Fracturing: Non-Uniform Distributions of Perforation Clusters that Promote Simultaneous Growth of Multiple Hydraulic Fractures
}

\author{
A.P. Peirce, University of British Columbia and A.P. Bunger, SPE, University of Pittsburgh
}

For submission to SPE Journal

\begin{abstract}
One of the important hurdles in horizontal well stimulation is the generation of hydraulic fractures (HFs) from all perforation clusters within a given stage despite the challenges posed by stress shadowing and reservoir variability. In this paper we use a newly-developed, fully coupled, parallel-planar 3D HF model to investigate the potential to minimize the negative impact of stress shadowing and thereby to promote more uniform fracture growth across an array of HFs by adjusting the location of the perforation clusters. In this model the HFs are assumed to evolve in an array of parallel planes with full 3D stress coupling while the constant fluid influx into the wellbore is dynamically partitioned to each fracture so that the wellbore pressure is the same throughout the array. The model confirms the phenomenon of inner fracture suppression due to stress shadowing when the perforation clusters are uniformly distributed. Indeed, the localization of the fracture growth to the outer fractures is so dominant that the total fractured area generated by uniform arrays is largely independent of the number of perforation clusters. However, numerical experiments indicate that certain non-uniform cluster spacings promote a profound improvement in the even development of fracture growth. Identifying this effect relies on this new model's ability to capture the full hydro-dynamical coupling between the simultaneously evolving HFs in their transition from radial to PKN-like geometries.
\end{abstract}

\section{Introduction}

Horizontal wells often have a significant proportion of non-producing perforation clusters (Miller and Waters 2011). One issue is the non-uniformity of reservoir properties, including the in-situ stress, along the well (e.g. Baihly et al. 2010, Cipolla et al 2011). Another issue is the well-known phenomenon known as "stress shadowing" that refers to suppression of some hydraulic fractures by the compressive stresses exerted on them by neighboring hydraulic fractures (e.g. Gemanovich et al. 1997, Fisher et al. 2004, Abass et al. 2009, Meyer and Bazan 2011). Using a fracture array model it has been recently shown that the competition between the stress shadow effect and the drive of the system to minimize the energy dissipated via viscous fluid flow leads to a stable spacing for contained hydraulic fractures (i.e. growing in length $L$ but with a fixed height $H$ ) that is $\sim 1.2-2.5$ times the height (Bunger et al. In Press). However, the fracture spacing used in practice is significantly smaller than that of these energy-minimizing configurations, which ensure stable fracture growth without localization. Indeed, most horizontal wells are fractured in stages that involve an array of perforation clusters distributed within a total zone length $Z$ that is usually similar to, or somewhat shorter than, the contained fracture height. Hence, while the work of Bunger et al. (In Press) is consistent with past observations of multiple hydraulic fracture growth from stages that are long relative to the fracture height (Fisher et al. 2004), it does not preclude the possibility that localization to 1 or 2 fractures might be inevitable due to stress shadowing when the zone length is similar to or less than the fracture height.

The issue of stress shadowing is, in a sense, more problematic than the issue of reservoir heterogeneity. Efforts to choose perforation cluster locations in an integrated way with reservoir characterization show some promising improvements (Cipolla et al. 2011). However, if localization due to stress shadowing is inevitable and uncontrollable by engineering design, then the uniformity of stimulation that can be achieved is fundamentally limited even for a mechanically homogeneous reservoir. It is therefore vital to address both potential sources of stimulation non-uniformity in a complimentary manner.

This study uses a newly-developed parallel-planar 3D hydraulic fracturing simulator that includes full coupling between fluid flow, fracture growth, and elastic deformation of the rock via the implicit level set algorithm developed by Peirce and Detournay (2008). The model expands on this prior work by including the non-local, fully 3D stress interaction among multiple hydraulic fractures. Furthermore, the total fluid injected into the wellbore is assumed to be constant while the fluid influx to each hydraulic fracture is partitioned dynamically in such a way that the wellbore pressure is constrained to be equal across all fractures in the array. This numerical approach is therefore distinct from so-called pseudo-3D models that rely on a local elasticity expression and therefore cannot appropriately track the growth of, and interaction among, the hydraulic fractures through the transition from radial to blade-like geometries, where, in the limit of zero height growth, the latter is approximated by the classical PKN solution (Perkins and Kern 1961, Nordgren 1972). The model is also distinct from models of multiple fracture growth that assume a uniform pressure within the fractures, a temporally constant pressure at the inlet, and/or uniform distribution of fluid among the fractures.

Because it is able to capture the fully-coupled mechanics including the radial to PKN-like transition with an energyminimizing partitioning of fluid, the new Planar3D simulator is appropriate for investigating fracture designs that promote simultaneous growth from multiple perforation clusters in a homogeneous reservoir. The model demonstrates that choosing specific, non-uniformly spaced perforation cluster locations can actually reduce the negative impact of stress shadowing in 
order to obtain more uniform hydraulic fracture growth. In fact, these numerical experiments demonstrate the existence of an unexpected regime of growth in which the stress-interaction between the hydraulic fractures seems to actually promote the uniformity of the growth rather than favoring the growth of some fractures while suppressing others. The result is a new, model-driven strategy for significantly reducing the number of non-producing perforation clusters through relatively straightforward and low-risk modifications to current best practice.

\section{Description of the numerical model}

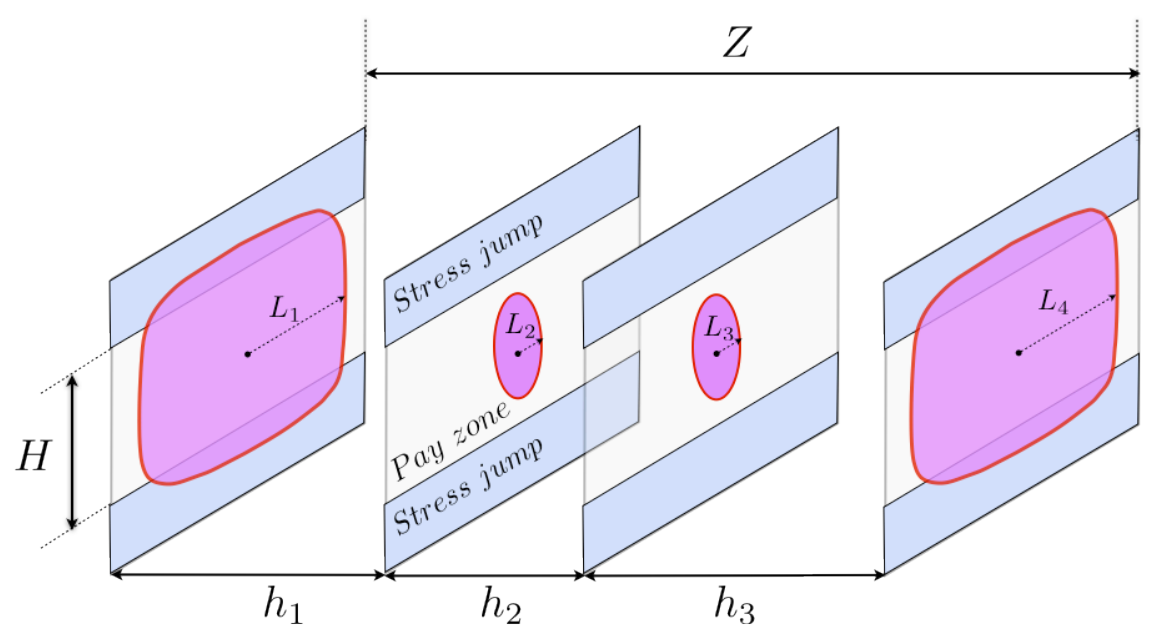

Fig. 1: Geometric configuration of the experimental perforation stage of length $Z$ within a pay zone of height $H$ in which $h_{k}$ is the fracture spacing.

\section{Geometric configuration:}

We consider an array of $N$ planar fractures to be distributed within a zone (i.e. one stage) of length $Z$ (see Fig. 1). The spacing $h_{k}, k=1, \ldots, N-1$ between each of the planes is such that:

$$
\sum_{k=1}^{N-1} h_{k}=Z
$$

The fractures are assumed to be initially radially symmetric and to be centered in the middle of a pay zone of height $H$, which is bounded by symmetrically distributed stress barriers across which the confining stress jumps from $\sigma_{z z}{ }^{0}$ to $\sigma_{z z}{ }^{0}+\Delta \sigma_{z z}{ }^{0}$. Once the fractures reach the stress barriers they transition from radial to PKN-like fractures with height growth, where the degree of finite height growth is limited by the increase in the confining stress field. Let $L_{k}$ denote the maximum distance from the center to the perimeter of the fracture in the $k$-th fracture plane, so that for a radial fracture $L_{k}$ corresponds to the fracture radius while for a PKN-like fracture $L_{k}$ corresponds to the fracture half-length. In the numerical experiments we consider the cases $N=5$, and 6 . For each of these $N$ values we vary the spacing $h_{k}$ between the planes in the array of fractures and measure the effect on the evolution of the footprints of the fractures within the planar array. For each such configuration the solutions are compared with those in which the same number of fracture planes $N$ are uniformly distributed within the array.

Note that by confining hydraulic fracture growth to a series of parallel planes we have assumed that the out-of-plane deflection of their paths is negligible. Of course this assumption must be carefully evaluated because the same stress shadow that can suppress growth of some fractures will also cause the paths of most, if not all, fractures in the array (depending on symmetry) to be deflected. Here we justify the planar assumption, albeit tentatively, based on a previous numerical, laboratory, and minethrough study showing that arrays of sequentially-placed hydraulic fractures under realistic reservoir conditions can grow with small enough deflection that it can usually be neglected at the relevant scale of the problem (Bunger et al. 2011, 2012, Kear et al. 2013). That being said, simultaneous growth is expected to produce more complicated interactions because of the ability for the growing fracture tips to interact (Sesetty and Ghassemi 2013), and the assumption of planarity embedded in the present model must therefore be examined in detail in future work.

\section{Assumptions of the model:}

The equations governing the propagation of a hydraulic fracture in a reservoir have to account for the dominant physical processes taking place during the treatment, namely the deformation of the rock, the creation of new fracture surfaces, the flow of the fracturing fluid in the crack. Besides the standard assumptions regarding the applicability of linear elastic fracture mechanics (LEFM) and lubrication theory, we make a series of simplifications that can readily be justified for the purposes of this contribution: (i) the rock is homogeneous and impermeable (the toughness $\mathrm{K}_{\mathrm{Ic}}$, Young's modulus E, and Poisson ratio $v$ 
thus have uniform values), (ii) the fracturing fluid is incompressible and Newtonian (with dynamic viscosity $\mu$ ), (iii) the fracture is always in limit equilibrium, that is, growing quasi-statically with the propagation condition exactly satisfied, (iv) gravity is neglected in the lubrication equation, and $(\mathrm{v})$ the fluid front coincides with the crack front, because the lag between the two fronts is negligible under typical high confinement conditions encountered in reservoirs.

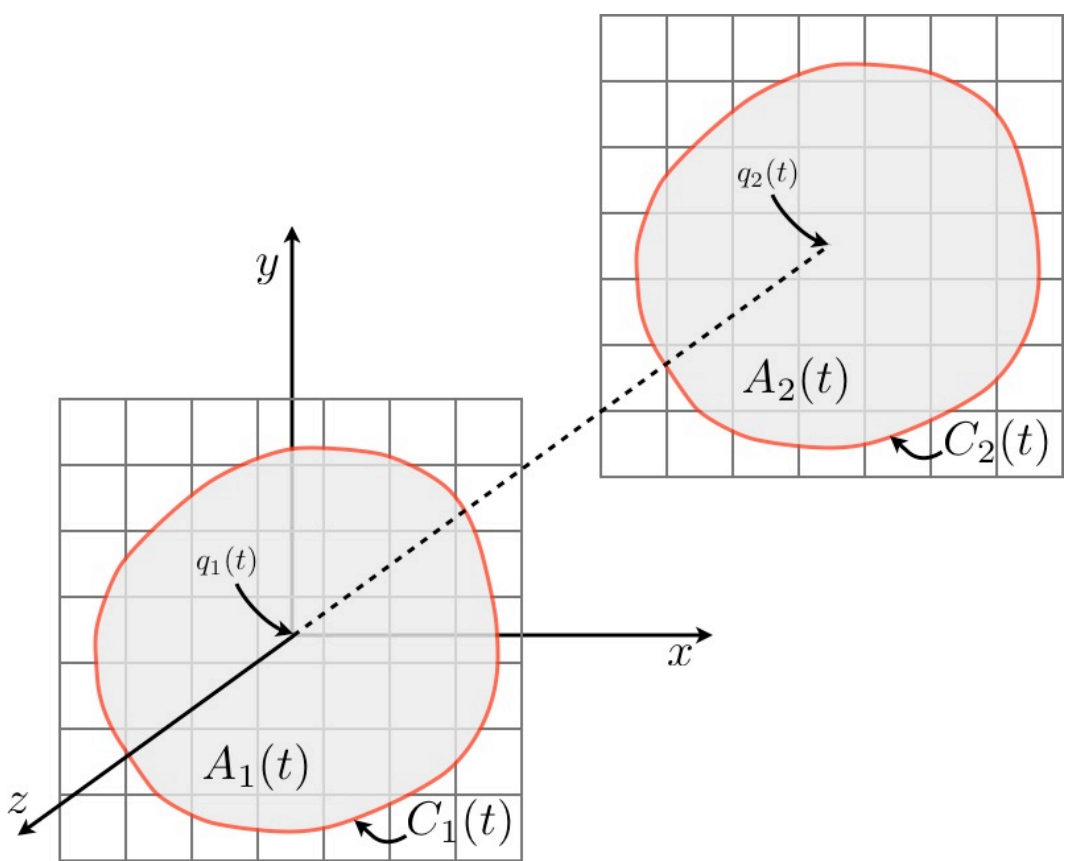

Fig. 2: Geometric configuration for the numerical model.

We assume that the fractures are constrained to grow in parallel planes that are perpendicular to the $z$ axis and defined by the equations $z=z_{k}, k=1, \ldots, N$, where $z_{k+1}=z_{k}+h_{k}$. The fracture in the $k$-th plane is assumed to occupy the region $A_{k}(t)$ enclosed within the boundary curve $C_{k}(t)$ and is driven by an influx of fluid $q_{k}(t)$ that is injected at the source point $\left(0,0, z_{k}\right)$. The volume of fluid $q_{k}(t)$ per unit time that is injected at each of the inlets is adjusted dynamically so that the sum of the fluid injected across the planar array equals the specified total volumetric injection rate $Q(t)$ and also such that the wellbore pressures $p^{b}{ }_{k}$ are constant across the entire array. These conditions are expressed as follows:

$$
\sum_{k=1}^{N} q_{k}(t)=Q(t), \quad p_{1}^{b}(t)=p_{2}^{b}(t)=\ldots=p_{N}^{b}(t)
$$

Eq. (2), then comprises a system of constraints and the actual partitioning of the influx and identification of the wellbore pressure.

\section{Elasticity equation:}

The infinite homogeneous elastic medium is assumed to be in a state of equilibrium and we use the displacement discontinuity formulation (see Crouch and Starfield, 1983) to express the $i$-th net stress component $\sigma_{i z}\left(x, y, z_{k}\right)$ on the $k$-th plane due to the displacement discontinuity components $D_{j}$ associated with the fracture footprints $A_{l}(t)$ in each of the $N$ planes as follows:

$$
\sigma_{i z}\left(x, y, z_{k}\right)=p_{f}(x, y)-\sigma_{i z}^{0}(x, y)=\sum_{l=1}^{N} \int_{A_{l}(t)} C_{i z j}\left(x-\chi, y-\eta, z_{k}-z_{l}\right) D_{j}\left(\chi, \eta, z_{l}\right) d \chi d \eta
$$

Here the indices $i$ and $j$ take on the values 1,2, and 3 corresponding to the $x, y$, and $z$ components, respectively, and $C_{i z j}$ represents the $i z$-th stress component at point $\left(x, y, z_{k}\right)$ due to a unit jump in the displacement field in the $j$-th direction at the point $\left(\chi, \eta, z_{l}\right)$. The fracture planes are assumed to be perpendicular to the minimum principal direction of the ambient geological stress field, to which the $z$-axis is aligned as shown in Fig. 2. The components of this geological stress field are represented by $\sigma_{i z}{ }^{0}$. Since the $z$-axis is a principal direction for the initial stress field, it follows that $\sigma_{x z}{ }^{0}=\sigma_{y z}{ }^{0}=0$, while $\sigma_{z z}{ }^{0}$ is the initial confining stress component. The fractures are assumed to be completely filled with fluid so that there is no fluidlag and the net normal stress within the rockmass throughout the fracture regions $A_{l}(t)$ must match the fluid pressure $p_{f}$ applied to the sides of the fracture. It follows, therefore, that the net stress components are defined by the conditions: 


$$
\sigma_{x z}\left(x, y, z_{k}\right)=0, \sigma_{y z}\left(x, y, z_{k}\right)=0, \sigma_{z z}\left(x, y, z_{k}\right)=p_{f}(x, y)-\sigma_{z z}^{0}(x, y)
$$

The three displacement discontinuity components $D_{j}$ comprise two shear (or ride) discontinuities $D_{x}$ and $D_{y}$, and a single normal displacement discontinuity component $D_{z}$, which represents the fracture width $w$ or crack opening displacement, i.e., $D_{z}\left(x, y, z_{l}\right)=w_{l}(x, y)$.

\section{Fluid flow equation:}

The applicable Reynolds lubrication equation, relating the fracture aperture $w_{l}(x, y)$ and corresponding fluid pressure $p_{f, l}$ within each fracture footprint $A_{l}(t)$, is obtained by combining the continuity equation with Poiseuille's law

$$
\frac{\partial w_{l}}{\partial t}=\frac{1}{\mu^{\prime}} \nabla \cdot\left(w_{l}^{3} \nabla p_{f, l}\right)+q_{l}(t) \delta(x, y), \text { for }(x, y) \in A_{l}(t)
$$

where $\mu^{`}=12 \mu$ is the scaled dynamic viscosity and $q_{l}(t)$ is the volumetric injection rate of fluid into the $l$-th fracture plane.

\section{Boundary and Propagation conditions:}

The coupled equations (2)-(5) are augmented by requiring that the fracture apertures are zero and that the fluid-fluxes vanish along the boundary curves $C_{l}(t)$, i.e.,

$$
\left.w_{l}\right|_{C_{l}(t)}=0, \text { and }\left.w_{l}^{3} \frac{\partial p_{f, l}}{\partial n}\right|_{C_{l}(t)}=0
$$

Given the locations of the fracture boundary curves $C_{l}(t)$, Eqs. (2)-(6) are sufficient to determine the aperture and fluid pressure fields $w_{l}(x, y)$ and $p_{f, l}$ in within each of the propagating fractures. In order to determine the location of each of the fracture boundaries $C_{l}(t)$ an additional propagation condition is required. If the elastic material has a non-zero toughness, the mode I stress intensity factor $K_{I \mathrm{I}}$ is assumed to be in limit equilibrium with the fracture toughness according to linear elastic fracture mechanics. This condition can be conveniently expressed by the following asymptotic relation (see Rice 1968)

$$
w_{l} \stackrel{s \rightarrow 0}{\sim} \frac{K^{\prime}}{E^{\prime}} s^{1 / 2}
$$

where $s$ is the normal distance to the fracture front and $K^{\prime}$ and $E^{\prime}$ are the scaled toughness and plane strain modulus defined by

$$
K^{\prime}=4\left(\frac{2}{\pi}\right)^{1 / 2} K_{I c} \text { and } E^{\prime}=\frac{E}{1-v^{2}}
$$

In the event that the fracture toughness is zero or negligible so that the fracture is propagating in the viscosity dominated regime, the appropriate tip asymptotic behavior is given by (see Desroches et al. 1994)

$$
w_{l} \sim 2^{1 / 3} 3^{5 / 6}\left(\frac{\mu^{\prime} V}{E^{\prime}}\right)^{1 / 3} s^{2 / 3}
$$

Here $V$ is the normal velocity of the fracture front $C_{l}(t)$.

\section{Discretization:}

In order to discretize the governing equations we follow the procedure used by Peirce and Detournay (2008). We consider a mesh comprising square elements that covers each of the regions within the designated planes into which the fractures are likely to grow (see Fig. 2). On the $m n$-th element of the $l$-th fracture plane, the fracture aperture is assumed to be piecewise constant, collocated at the center of the element, and represented by the average value $w_{l, m n}$ over the element. By integrating the Reynolds lubrication equation over the square elements in each of the fractures, a finite volume discretization is defined with respect to quantities sampled at the element centers. At the peripheries of each of the fractures the boundary curves $C_{l}(t)$, which are typically irregular and may not conform to the structured square mesh, are represented by using a concept of partially filled tip elements in which quantities are also sampled at element centers.

\section{The Implicit Level Set Algorithm (ILSA) to locate the fracture boundary:}

The fracture free boundary within each plane is located by means of the ILSA scheme (see Peirce and Detournay 2008) in which the asymptotic expansion for the fracture width in the vicinity of the fracture perimeter (such as those given in (7) and (9)) is inverted in order to determine the shortest distance to the fracture boundary. The following iterative process is then used within each fracture plane: given an initial guess for the fracture boundary $C_{l}(t)$, determine the corresponding trial fracture width $w_{l, m n}$ and the corresponding fluid pressure $p_{f, l, m n}$ for every element $m n$ whose center falls within $C_{l}(t)$; within each fracture use the trial width values within the ribbon of elements, which are completely filled with fluid and which share 
at least one side with a partially filled tip element, to estimate the distance to the free boundary by inverting the applicable tip asymptotic behavior; use these estimates of the distance to the free boundary as initial conditions for the eikonal equations

$$
|\nabla T(x, y)|=1
$$

With these initial conditions, the level set curves $T_{l}(x, y)=0$ of the solutions to Eq. (10) provide new estimates of the locations of the fracture boundaries. The fracture boundary curves $C_{l}(t)$ are then moved to coincide with the curves $T_{l}(x, y)=0$ and the iterative process is repeated until convergence is achieved.

\section{Numerical Experiments}

The numerical results are mainly impacted by the number of fractures, the ratio of the zone length to the fracture height $Z / H$, and by the relative importance of energy dissipation associated with rock breakage versus viscous fluid flow. To illustrate the phenomenon we consider the cases $N=5$ and $N=6$ with $Z / H=1$ and where viscous dissipation dominates rock breakage. The latter assumption can be considered valid, at least from the perspective of radially-growing hydraulic fractures, as long as the treatment time satisfies (e.g. Detournay 2004)

$$
t_{T O T}=\left(\frac{\mu^{\prime} Q_{o}^{3} E^{\prime}}{K^{\prime 18}}\right)^{1 / 2}
$$

Within these constraints, and representative of other parameter choices satisfying the same constraints, the following parameters are chosen for convenience

$$
\begin{aligned}
& E=9.5 \mathrm{GPa}, \quad v=0.2, \quad K_{I c}=0 \mathrm{MPa} \cdot \mathrm{m}^{1 / 2} \\
& \mu=1 \mathrm{~Pa} \cdot \mathrm{s}, \quad Q_{0}=0.1 \mathrm{~m}^{3} / \mathrm{s}, \quad Z=20 \mathrm{~m} \\
& \sigma_{z z}^{0}=70 \mathrm{MPa}, \quad \Delta \sigma_{z z}^{0}=5 \mathrm{MPa}, \quad H=20 \mathrm{~m}
\end{aligned}
$$

In the results given below all lengths and widths will be given in $m$, time given in seconds, and pressures are given in $P a$ and the numbering assigned to the planes starts with one of the outer planes. Furthermore, as a measure for the effectiveness of a given configuration at generating fractured surface area, we define the quantity $\mathrm{A}(t)$ to be the total area in $m^{2}$ of all the footprints in the perforated planes, i.e.,

$$
A(t)=\sum_{l=1}^{N} \int_{A_{l}(t)} d \chi d \eta
$$

\section{Odd number of perforations $N=5$ :}

\section{Uniform array:}

Firstly, we consider the case where the perforations are uniformly spaced so that $h_{k}=5 \mathrm{~m}$ for $k=1, \ldots, 4$ and the fracture planes have $z$ coordinates $z_{1}=0, z_{2}=5, z_{3}=10, z_{4}=15$, and $z_{5}=20$. In Fig. 3 we provide perspective snap-shots at times $t=35,71,108$, and 143 seconds. The fracture footprints are colored with the magnitudes of the local fracture width fields whose color bar is depicted on the right of each plot (in meters). All the fractures initiate in the radial geometry with an initial radius of $2.38 \mathrm{~m}$. Radial growth proceeds in all fractures until some of them encounter the stress barrier, at which point they transition to bladelike geometries. Due to the mutual stress interaction among the five fractures that are competing to grow, they do not develop at the same rate. Instead the fracture growth in the array is rapidly localized in the two outer fractures while growth of the inner fractures is stunted. This localization of growth in the outer fractures is to be expected because they have no constraint on their growth from outside the array. In contrast, the interior fractures have to compete with one another in an induced compressive stress field that is established by the outer fractures and enhanced by any additional growth by the interior fractures. This phenomenon, in which there is localization of the growth in the two outer fractures and stunted growth of the inner fractures, is known as "stress-shadowing" (e.g. Gemanovich et al. 1997, Fisher et al. 2004, Abass et al. 2009, Meyer and Bazan 2011). The localization becomes more pronounced with time while growth of the inner fractures is minimal. Note that the central fracture is suppressed in the same way when an array of $N=3$ fractures is considered, as we shall see below.

The dominance of the outer fractures can be further appreciated by tracking the evolution of their length, width, and portion of the fluid flux relative to the interior fractures. Fig. 4 shows time evolution plots of these quantities, along with the inlet pressure, for each of the fractures in the array. Hence we show results for fracture 1 (which is identical to fracture 5), 2 (which is identical to fracture 4), and fracture 3. Moreover, in all the time traces plotted below we adopt the following colorcoding/line style convention: red/solid for fracture 1, green/dashed for fracture 2, and blue/dash-dot for fracture 3. In Fig. 4 (a) we plot the time evolution of the ratio of the fracture diameter/length to the height of the pay zone. This ratio $2 L_{/} / H$ reaches a value of 1 at the transition from radial to PKN-like fracture propagation. From this plot it can be seen that the outer fractures, 1 and 5 (depicted by the red/solid line), rapidly transition from radial to PKN-like geometries, while the inner 
fractures never move out of the radial regime because they do not grow sufficiently to be impacted by the height barrier. In Fig. 4 (b) we observe that shortly after initiation, fractures 1 and 5 consume all of the injected fluid. In Fig. 4 (c) the signature of the transition from radial to PKN-like propagation of fractures 1 and 5 can be clearly seen from the change in curvature in the well-bore width evolution plot. Consistent with the second constraint imposed in Eq. (2), from Fig. 4 (d) we observe that the well-bore pressures are the same for all the fractures. This pressure decreases monotonically as time evolves with a net pressure in the range of 3-8 $\mathrm{MPa}$.

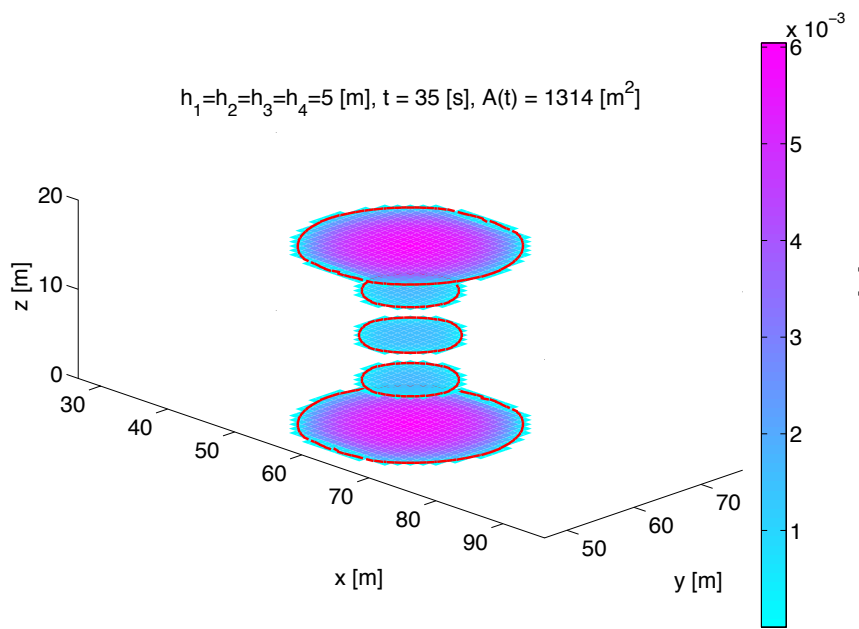

(a)

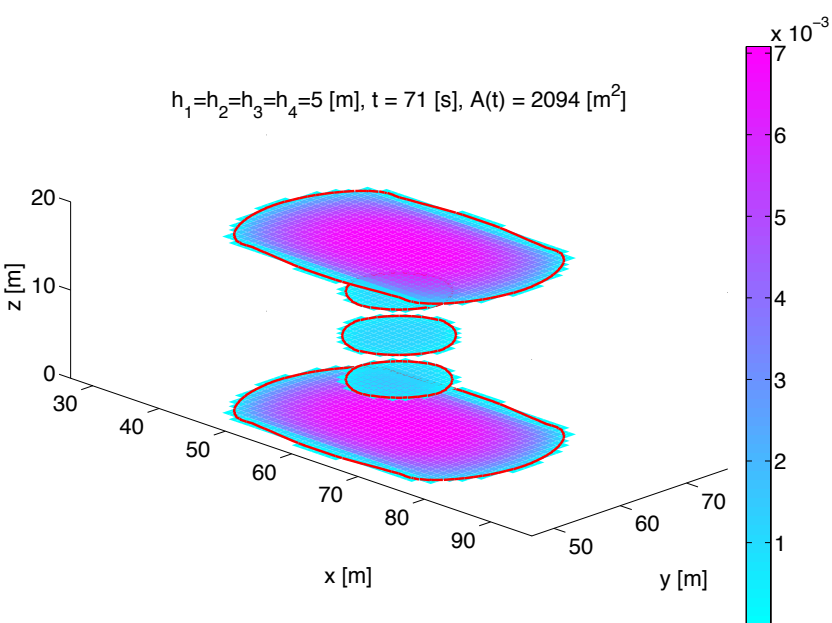

(b)

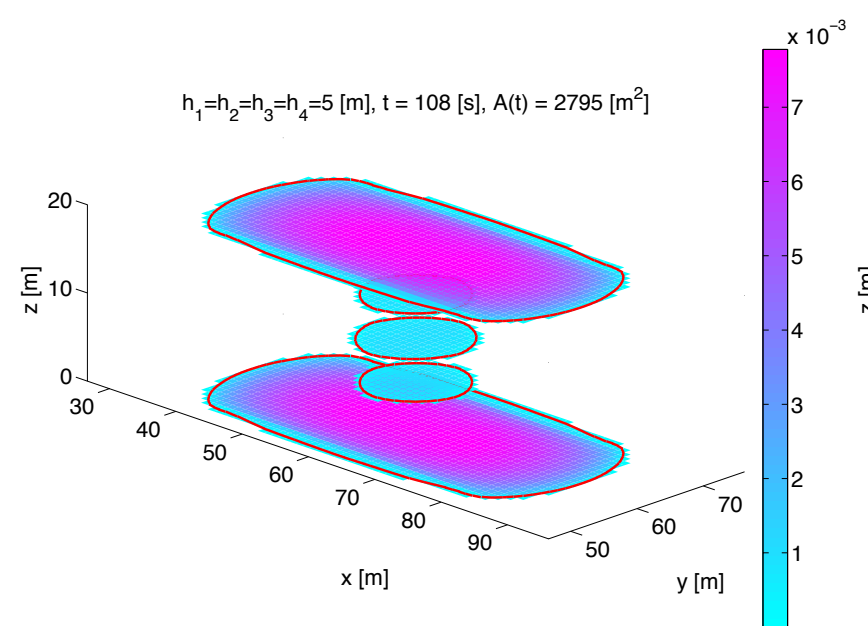

(c)

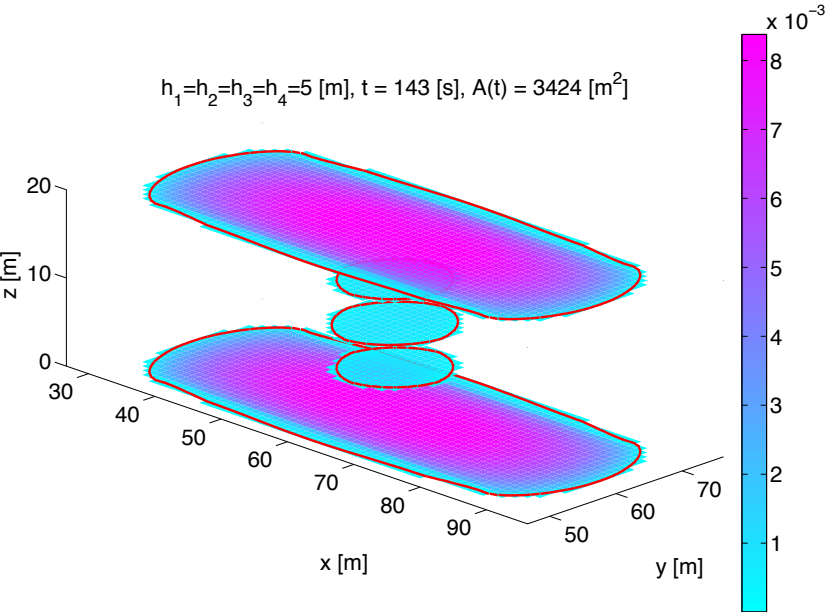

(d)

Fig.3: Perspective views of snap-shots of the five footprints in the uniform array at different times. The footprints are colored by the width field $w_{l}(x, y)$ for which the color bars are given in $\mathrm{m}$. 

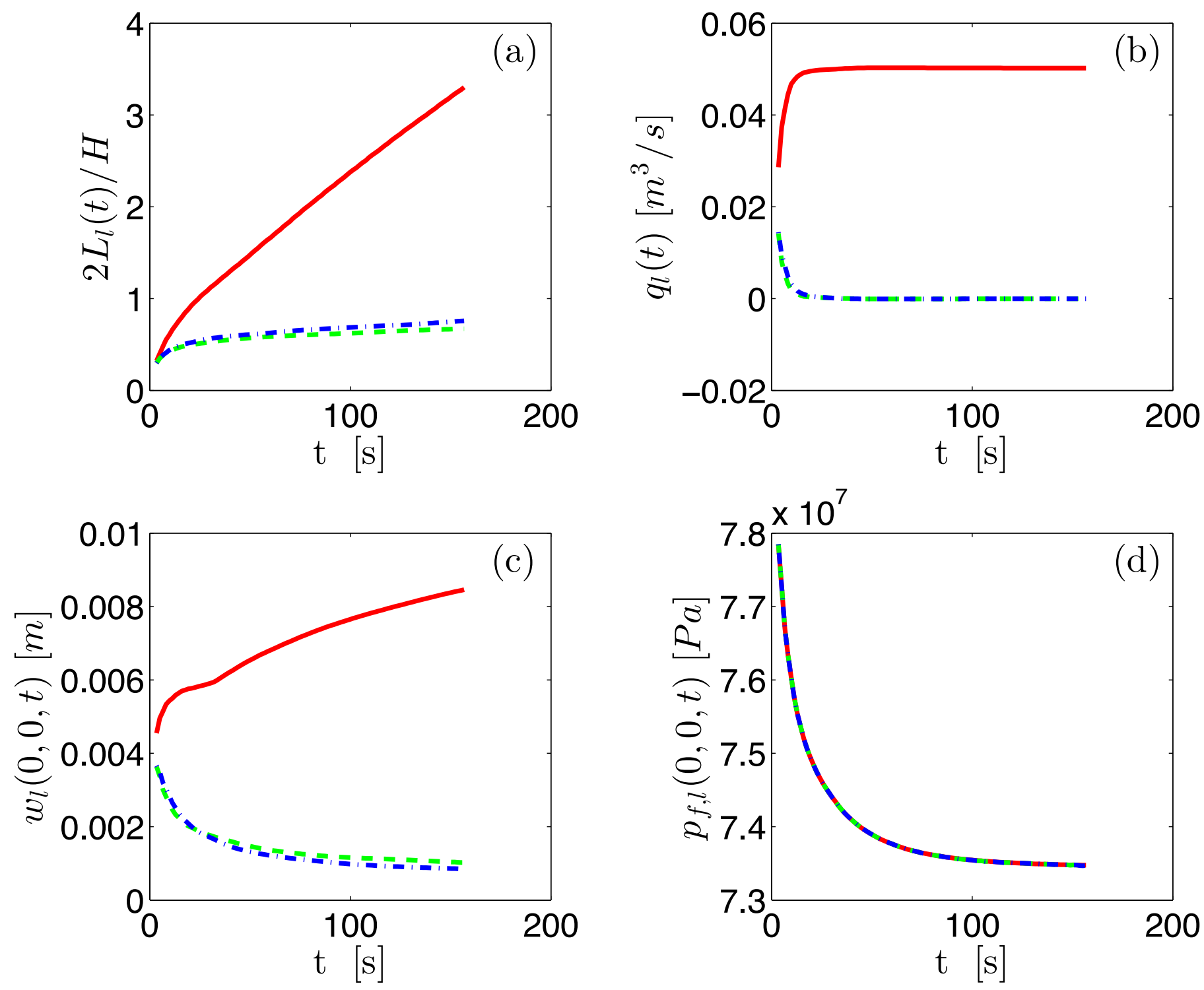

Fig. 4: Time evolution of $2 L_{l} / H, q_{l}$, and the well bore widths $w_{l}(0,0, t)$ and fluid pressures $p_{f, l}(0,0, t)$ for $N=5$ uniformly distributed fractures. Red/solid lines are used for the fractures in planes $1 \& 5$, green/dashed lines for the fractures in planes $2 \& 4$, and blue/dash-dot lines for the fracture in plane 3 .

\section{Nonuniform array:}

We now introduce a modification to the uniform array in which fractures 2 and 4 are moved so that $h_{1}=3.5, h_{2}=6.5, h_{3}=6.5$, and $h_{4}=3.5$, corresponding to fracture planes having $z$ coordinates $z_{1}=0, z_{2}=3.5, z_{3}=10, z_{4}=16.5$, and $z_{5}=20$. Note that this particular spacing was specifically chosen through trial and error because it most strongly exhibits the phenomenon that will be discussed.

Fig. 5 shows perspective snap-shots of these nonuniformly distributed fractures at the times $t=35,71,108$, and 143 seconds. The fracture footprints are colored with the magnitudes of the local fracture width fields whose color bar is depicted on the right of each plot. The first snapshot (Fig. 5 (a)) corresponds to a point where all fractures are still radial. However, unlike the uniform spacing case (Fig. 3(a)), where fractures 1 and 5 grew preferentially and fractures 2-4 were suppressed, Fig. 5(a) shows that fractures 1,3 , and 5 have all grown to approximately the same radius and only the two interference fractures (2 and 4) are showing inhibited growth. The contrast is also apparent in the evolution of the fracture lengths shown in Fig. 4(a) for the uniform spacing and its counterpart for the nonuniform spacing, Fig. 7(a).

When the total length of fractures 1,3 , and 5 is between 1 and 1.5 times the fracture height, i.e. just as the radial to PKN-like transition is commencing, a new phase is reached in which the role of the interference fractures switches from being passive and accepting relatively little fluid to accepting the majority of the fluid and actively driving the dynamics of the fracture development throughout the array. This transition is apparent from the change in the evolution of the influx to fractures 2 and 
4 that occurs just after $\mathrm{t}=50$ seconds in Fig. 7(b). Fig. 5 (b) shows the fracture footprints just after this transition time $t_{c}$ between these two regimes. The increased uptake of fluid in the still-radial interference fractures also has a suppressing effect on width near the well-bores for fractures 1 and 5. This suppression effect later also starts to affect fracture 3 and ultimately starts to choke further uptake of fluid into fractures 1,3 , and 5.

Further fracture growth in these choked fractures, 1, 3, and 5, is driven by a somewhat surprising mechanism. The fluid that was in these fractures in the region near the wellbore is displaced toward the perimeter as they are subjected to the induced stress associated with the now rapidly inflating fractures 2 and 4 . This outward squeezing of the fluid has the effect of advancing the fracture by the displacing the fluid from the vicinity of the wellbore rather than by influx from the wellbore. Concomitant with the onset of this second, "squeezing" phase of growth, the wellbore pressure increases by a few hundred $\mathrm{kPa}$ relative to the $\sim 5 \mathrm{MPa}$ net pressure (Fig. $7(\mathrm{~d})$ ), reaching a maximum at approximately the same time that the wellbore width of fractures 1,3 , and 5 begins to decline.

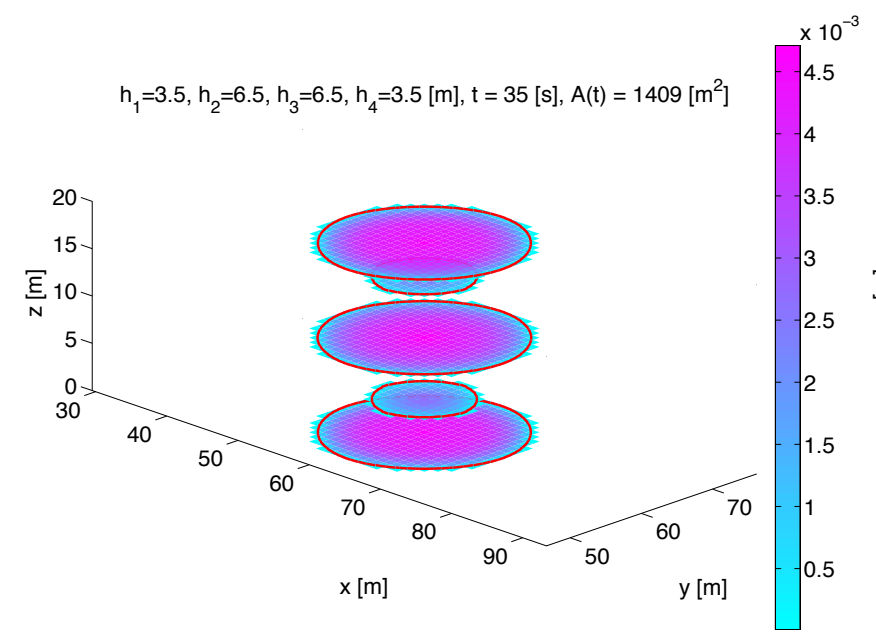

(a)

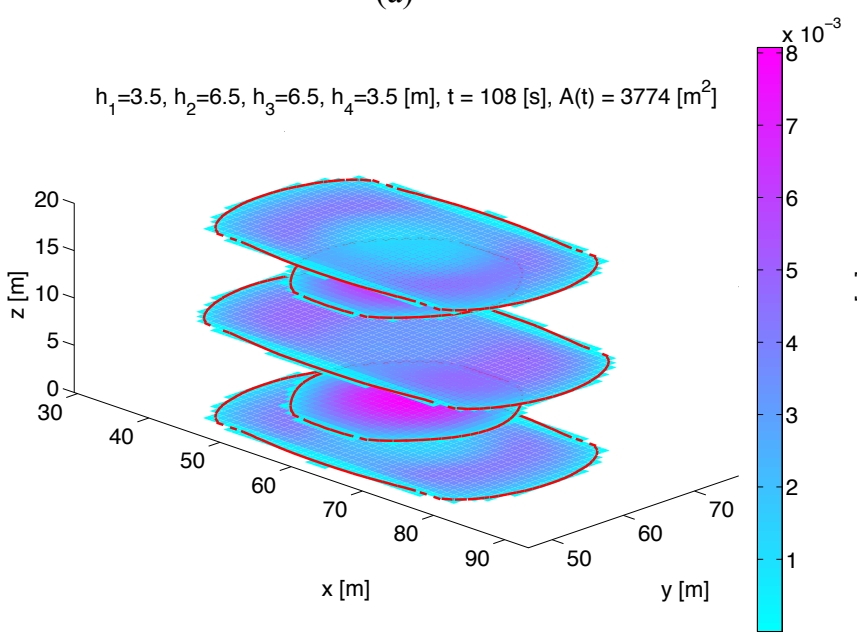

(c)

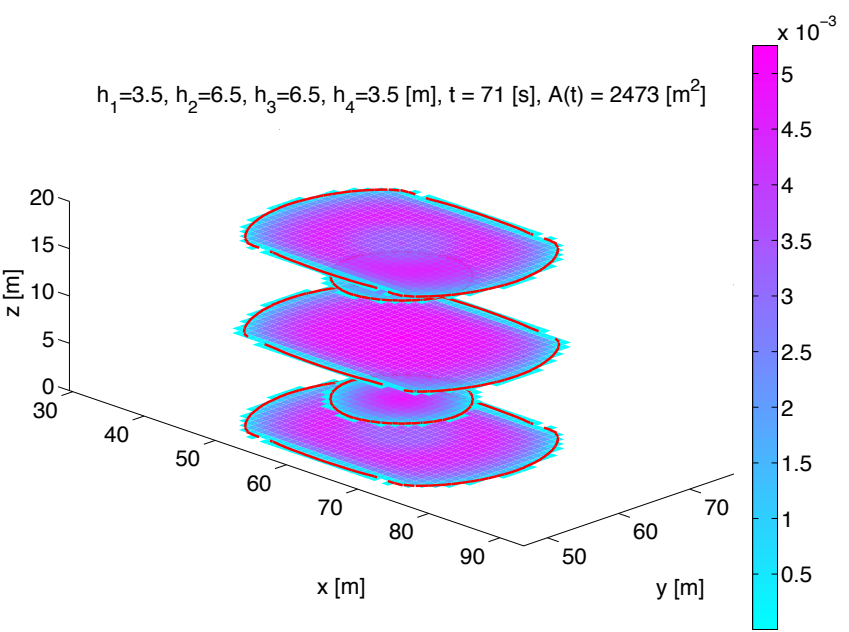

(b)

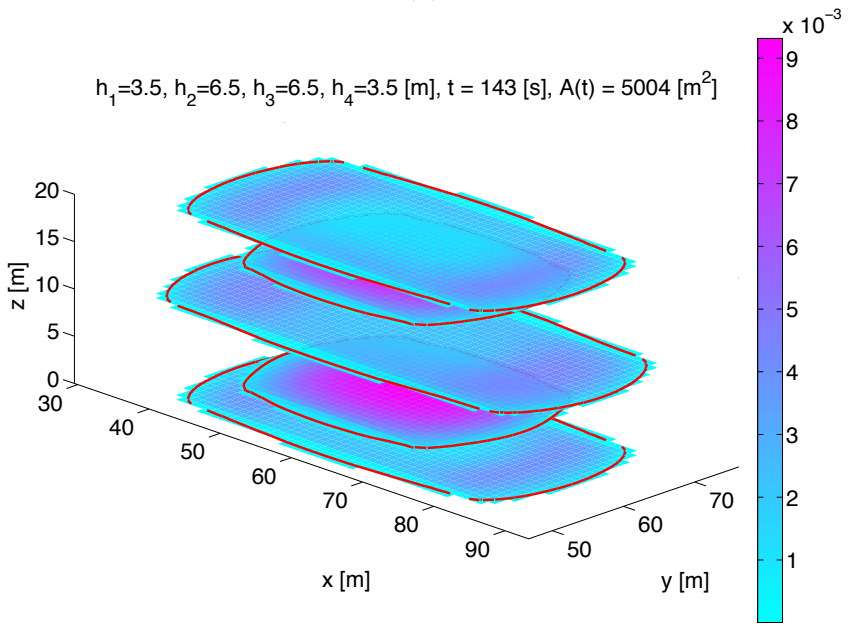

(d)

Fig.5: Perspective views of snap-shots of the five footprints in the non-uniform array at the same times as those for the uniform array presented in Fig. 3 . Each of the footprints are colored by the width field $w_{l}(x, y)$ for which the color bars are given in $\mathrm{m}$.

As growth continues, Fig. 5 (c) shows that by $t=108$ seconds the interference fractures have also transitioned from the radial to the PKN-like propagation regime. On the other hand, fractures 1, 3, and 5 have enlarged their footprints, mainly through squeezing-induced growth that has resulted in significant width reduction (Fig. 7(c)). By this point the pressure is in its second period of decline (Fig. 7(d)). From here on, the interference fractures, 2 and 4, take essentially all of the influx (Fig. 7(b)) and grow preferentially to eventually approach a similar size to fractures 1, 3, and 5 (Fig. 5(d)).

Since precisely the same injection rate and total volume was used for both the uniform and nonuniform simulations, Figs. 3 and 5 represent the two very different outcomes caused only by the relative location of fractures 2 and 4 . Perhaps most strikingly, the total fractured area generated by the nonuniform array compared to the uniform array is: $7 \%$ after $t=35 \mathrm{~s}, 22 \%$ after $t=71 \mathrm{~s}, 35 \%$ after $t=108 \mathrm{~s}$, and $46 \%$ after $t=143 \mathrm{~s}$. 
In order to better understand the role that the two interference fractures play in the dynamics of the fracture evolution in the nonuniform array, we compare the results of the five fracture array with those of a uniformly distributed three fracture array. In Fig. 6 we plot the perspective snap-shot for the three fracture footprints sampled at $\mathrm{t}=108$ seconds, which corresponds to the time at which the five fracture footprints are plotted in Fig. 3(c). Comparing these figures, it can be seen that the middle fracture in the 3 fracture array is subject to less constraint from the other growing fractures in the array than the middle fracture in the uniform 5 fracture array. However, it is still susceptible to stress shadowing and has a much smaller fracture width field than the outer fractures. Moreover, the total fractured areas for the $N=3$ and $N=5$, uniformly-spaced arrays is roughly similar, which indicates that the dominant fracture growth is localized in the outer fractures and the stress shadowing renders both arrays roughly equivalent in their ability to generate new fracture area from the interior fractures. Comparing Figs. 5 (b) and 6 it can be seen that the presence of the interference fractures makes it possible for the middle fracture in the 5 fracture array to develop at roughly the same rate as the outer fractures and is already more developed than the middle fracture in the 3 fracture array in spite of the fact that it has had roughly 34\% less time to grow. Comparing Figs. 5 (c) and 6 , which are sampled at the same time, it can be seen that even the interference fractures, which are the smallest in the 5 fracture array, are already more developed than the middle fracture in the 3 fracture array.

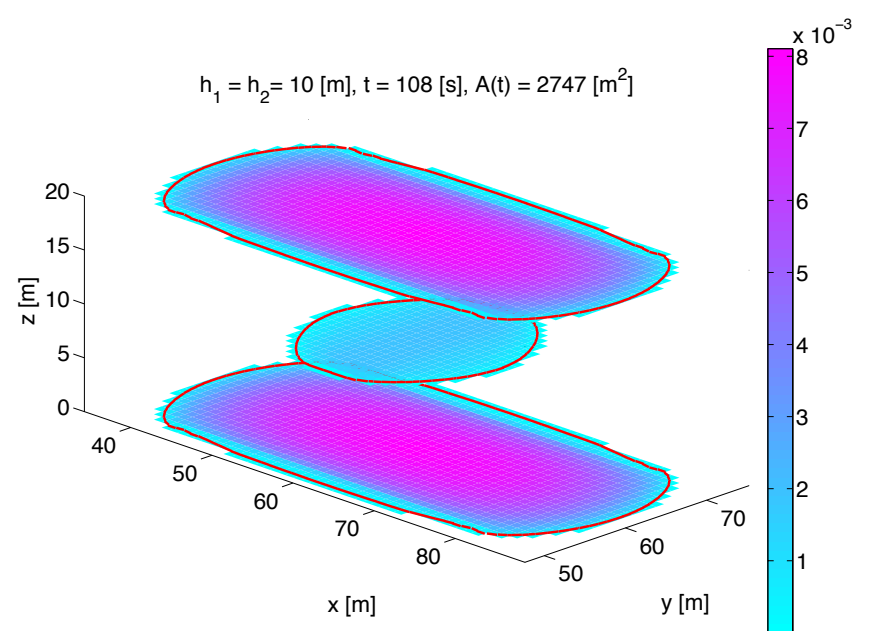

Fig.6: Perspective view of a snap-shot of footprints in the three-fracture array. The footprints are colored by the width field $w_{l}(x, y)$ for which the color bars are given in $\mathrm{m}$.

To summarize, we observe that a relatively minor, although specifically chosen, perturbation to the symmetry of the distribution of the perforations leads to a dramatically different development of the fractures in the array. Placing what we call the interference fractures 2 and 4 closer to the two outer fractures, 1 and 5 respectively, has the effect of impeding the runaway localization of growth in the outer fractures that was previously observed for the uniform array (Figs. 3 and 4 ). This enhanced competition between fractures 2-1 and 4-5 also reduces the constraint on fracture 3, which is observed to evolve at roughly the same rate as the outer fractures. 

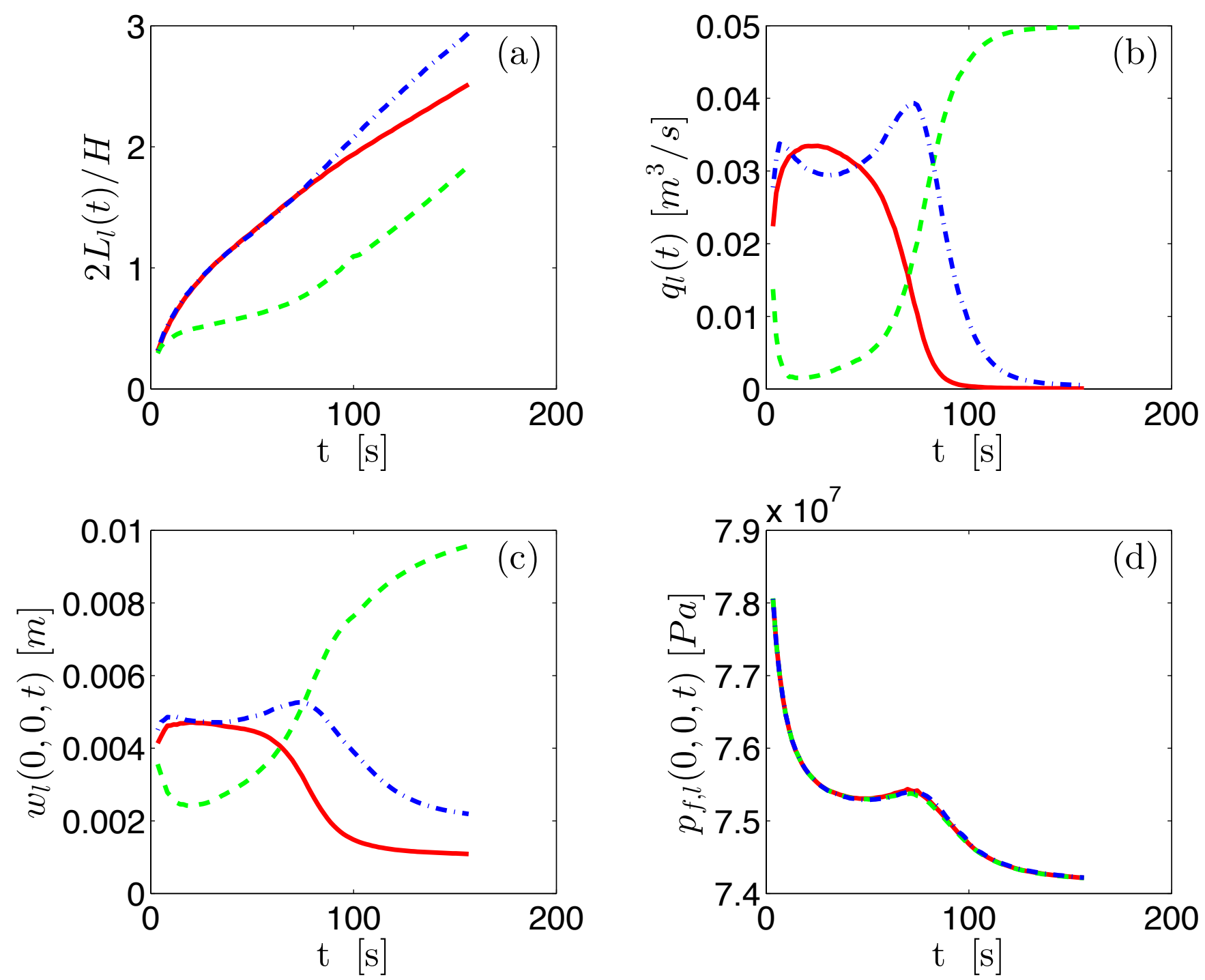

Fig. 7: Time evolution of $2 L_{l} / H, q_{l}$, and the well bore widths $w_{l}(0,0, t)$ and fluid pressures $p_{f, l}(0,0, t)$ for $N=5$ and $h_{1}=3.5, h_{2}=6.5, h_{3}=6.5, h_{4}=3.5$. Red $/$ solid lines are used for the fractures in planes $1 \& 5$, green/dashed lines for the fractures in planes $2 \& 4$ and blue/dash-dot lines for the fracture in plane 3.

\section{Even number of perforations $N=6$ :}

\section{Uniform array:}

Firstly, we consider the case with uniform spacing in which $h_{k}=4 m$ for $k=1, \ldots, 5$ so that the six fracture planes have $z$ coordinates $z_{1}=0, z_{2}=4, z_{3}=8, z_{4}=12, z_{5}=16$, and $z_{6}=20$. In Fig. 8, we provide perspective snap-shots of the six growing fractures for the uniform array at the times $t=35,71,107$, and 142 seconds. As with the $N=5$ case, the dominant growth of the two outer fractures and the stress shadowing of the interior fractures can be clearly seen. We observe that the total fractured area $A=3361 \mathrm{~m}^{2}$ of the uniform 6 fracture array is almost the same as the $3424 \mathrm{~m}^{2}$ generated by the uniform five-fracture array shown in Fig. 3 (d) when sampled at approximately the same time $t=142$ seconds while injecting at the same rate. This correspondence applies throughout the evolution of the fractures for these two uniform arrays. This further confirms that the dominant mechanism for generating new fracture area in a uniform array is through localization in the two outer fractures. Thus the interior fractures, which are subject to stress shadowing, are of little consequence when it comes to generating new fracture area. This results in roughly similar performance for all uniform arrays no matter how many fractures $N$ they may contain. The suppression of the interior fractures by the outer fractures is once again evident (Fig. 9) in the evolution of the length, width, and influx for fractures 1 (the same as 6), 2 (the same as 5), and 3 (the same as 4). In fact, these quantities evolve almost identically to the $N=5$ case (Fig. 4). Also, similar to the $N=5$ case, the wellbore pressure declines monotonically, although it is on the order of $100 \mathrm{kPa}$ higher relative to $\sim 3-4 \mathrm{MPa}$ net pressure for $N=6 \mathrm{case}$ (Fig 9 (d)). 


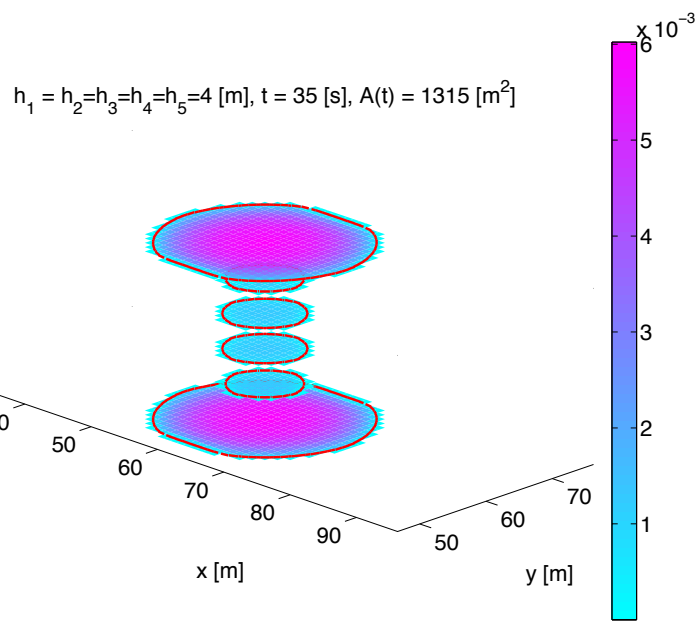

(a)

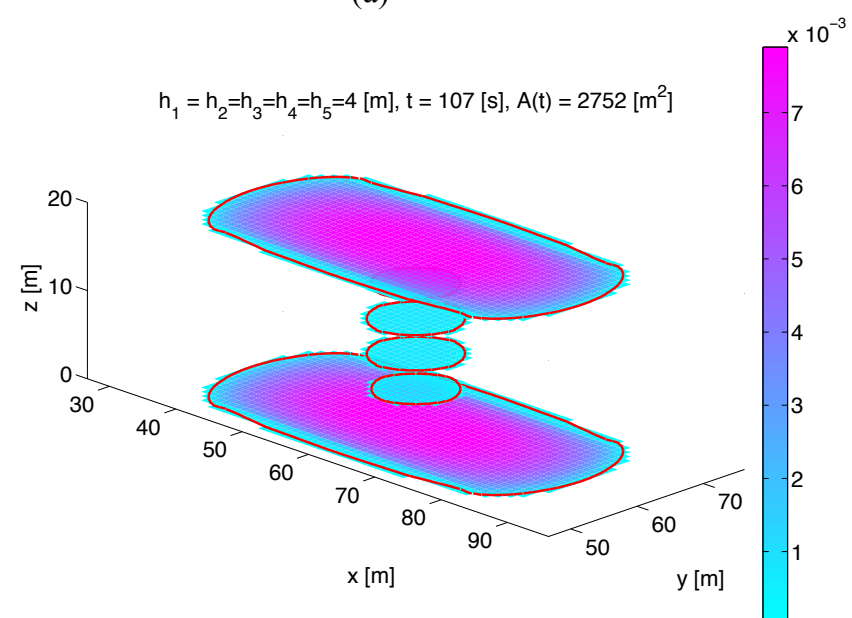

(c)

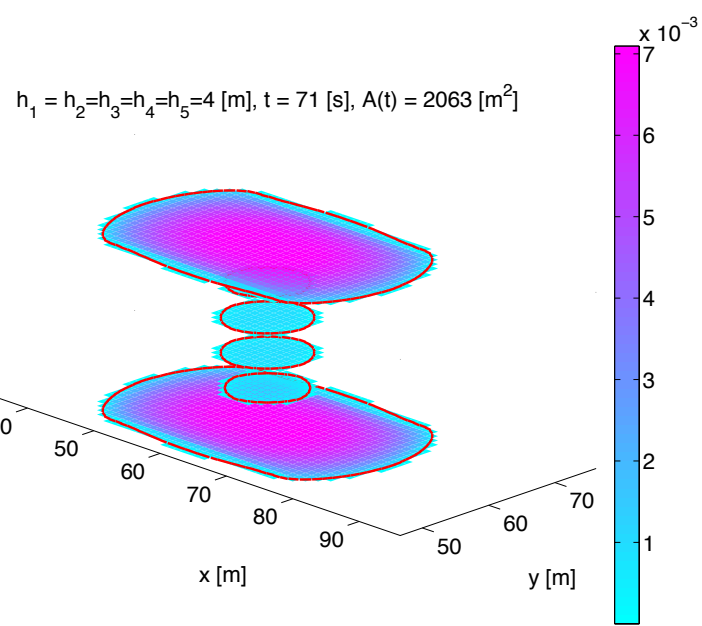

(b)

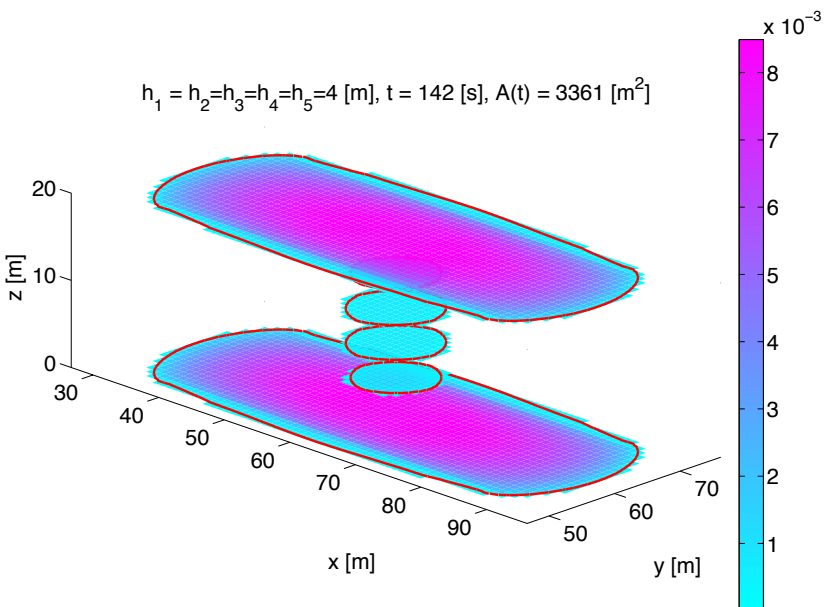

(d)

Fig.8: Perspective views of snap-shots of the six footprints in the uniform array at different times. The footprints are colored by the width field $w_{l}(x, y)$ for which the color bars are given in $\mathrm{m}$. 

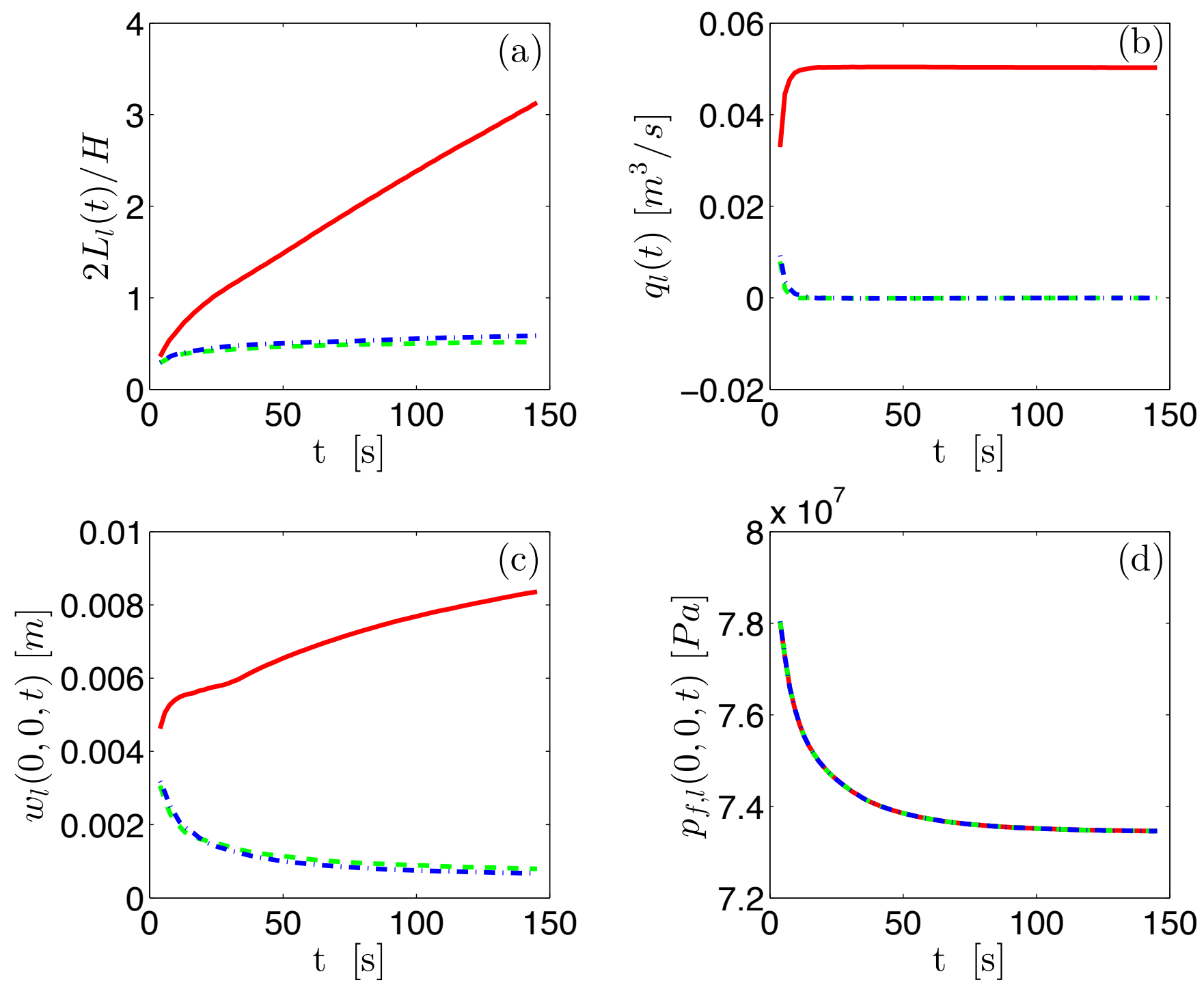

Fig. 9: Time evolution of $2 L_{l} / H, q_{l}$, and the well bore widths $w_{l}(0,0, t)$ and fluid pressures $p_{f, l}(0,0, t)$ for $N=6$ uniformly distributed fractures. Red/solid lines are used for the fractures in planes $1 \& 6$, green/dashed lines for the fractures in planes $2 \& 5$ and blue/dash-dot lines for the fractures in planes $3 \& 4$.

\section{Nonuniform array:}

For this experiment we introduce symmetric perturbations to the four inner planes in such a way that the pair 2 and 5 are the same distance from the center of the array $z=10$ but on opposite sides and similarly the pair 3 and 4 are the same distance from the center of the array but on opposite sides. Indeed, we introduce perturbations to the uniform array in which fractures $k=2,3,4$, and 5 are moved so that $h_{1}=2.5, h_{2}=3.5, h_{3}=8, h_{4}=3.5$, and $h_{5}=2.5$ corresponding to fracture planes having $z$ coordinates $z_{1}=0, z_{2}=2.5, z_{3}=6, z_{4}=14, z_{5}=17.5$, and, $z_{6}=20$. Once again, this geometry was chosen because it was found to maximize the phenomenon that will be discussed.

In Fig. 10 we provide perspective snap-shots of the six, nonuniformly spaced hydraulic fractures at the times $t=35,71,107$, and 142 seconds. We observe that choosing this particular array spacing leads to dramatically different development of the fractures in the array compared to the uniform spacing case. The evolution begins with a period of approximately equal growth of fractures $1,3,4$, and 6 . This period ends when the length of these dominant fractures just exceeds the height and thus the geometry transitions to PKN-like growth. This first transition occurs just after the snapshot in Fig. 10(a) and is evidenced by the sudden drop in influx to these initially dominant fractures, Fig. 11(b), earlier than for the $N=5$ array at about $t=40$ seconds, and the sudden increase in the influx to the interference fractures 2 and 5 . As was observed for the non-uniform $N=5$ array, the inflation and extension of the interference fractures suppresses the widths near the wellbores of the initiallydominant fractures (Fig. 11(c)) as the fluid that was in this region near the well-bore is displaced toward the perimeter and advances the fracture length in spite of there being nearly zero influx to these four fractures. The onset of the dominance of the interference fractures and squeezing of the initially dominant fractures corresponds to a short period of increase in the wellbore pressure (Fig. 11(d)). 
The result of this squeezing stage of growth can be seen in Fig. 10 (b), which shows that by $t=71 \mathrm{~s}$ the interference fractures are just about to transition from the radial to the PKN-like propagation regimes while the other fractures 1, 3, 4, and 6 have entered the PKN-like propagation regime and enlarged their footprints but have also thinned significantly. In Fig. 10 (c) it can be seen that by $t=107 \mathrm{~s}$ the interference fractures are just starting to overtake the other fractures while the compressive stresses they induce on the other fractures in the array has significantly slowed their growth. The wellbore pressure declines during this period and for the rest of the numerical experiment (Fig. 11(d)). Fig. 10 (d) shows that growth in the interference fractures ( 2 and 5) eventually becomes completely dominant; they have effectively halted any further growth in the other fractures in the array.

Comparing the total fractured areas $A(t)$ given in Figs. 8 and 10, we observe that the increase in total fractured area generated by the nonuniform array compared to the uniform array is: $17 \%$ after $t=35 \mathrm{~s}, 57 \%$ after $t=71 \mathrm{~s}, 68 \%$ after $t=107 \mathrm{~s}$, and $74 \%$ after $t=142 \mathrm{~s}$. Furthermore, since precisely the same total pump rates were used for the $N=5$ and $N=6$ arrays, it is useful to compare the total fracture area $A$ between the two arrays at the end of the experiment at approximately the same time. The six-fracture array achieves a total fracture area $A=5850 \mathrm{~m}^{2}$ at time $t=142 \mathrm{~s}$ compared to a total fracture area $A=5002 \mathrm{~m}^{2}$ attained by the five-fracture array at time $\mathrm{t}=141 \mathrm{~s}$.

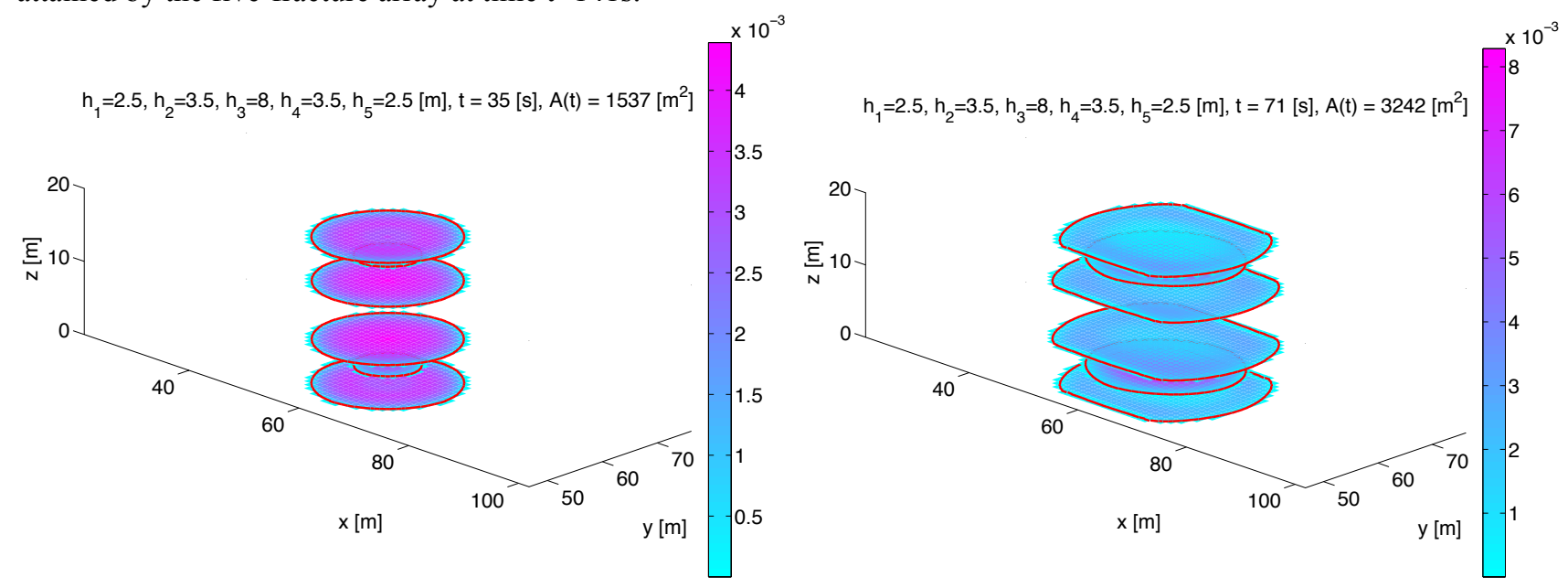

(a)

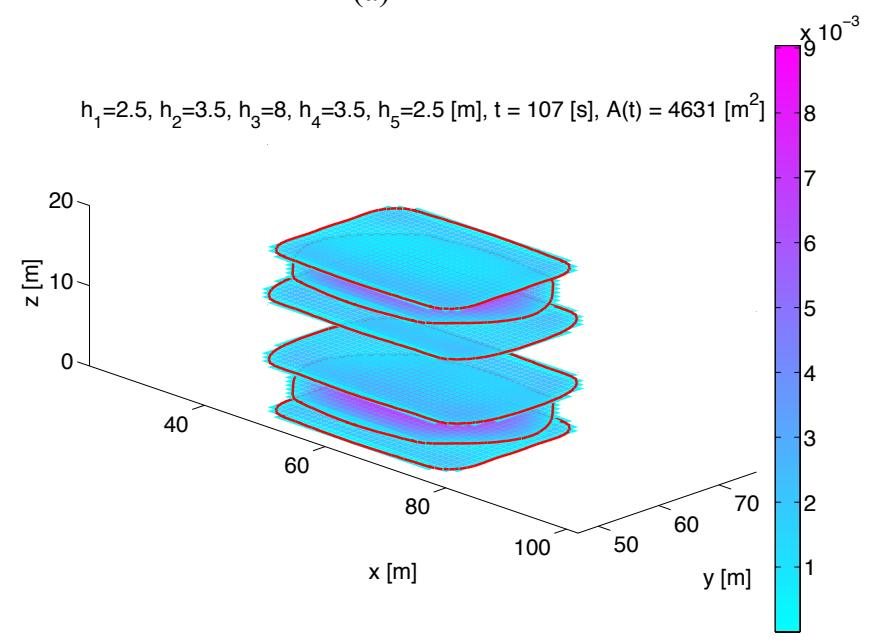

(c) (b)

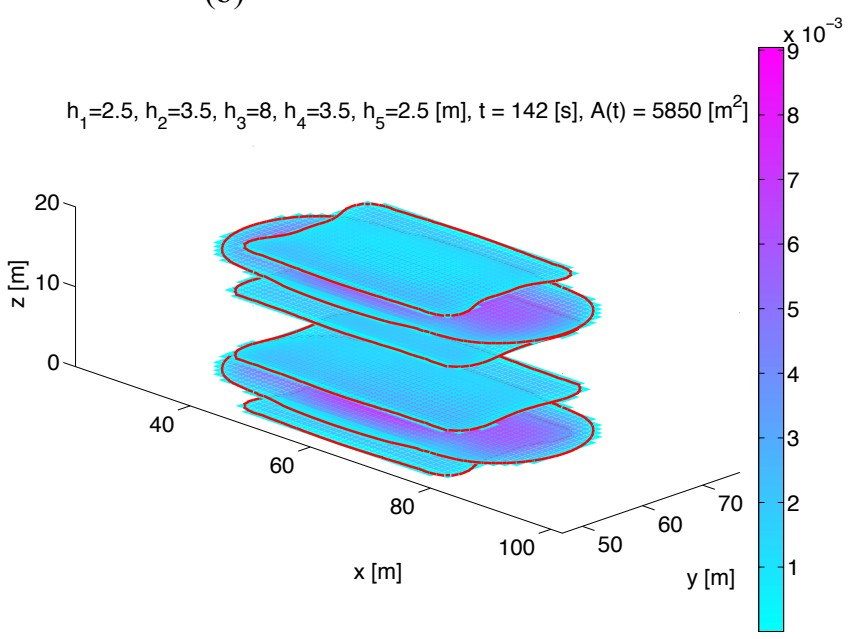

(d)

Fig. 10: Perspective views of snap-shots of the six footprints in the non-uniform array at different times. The footprints are colored by the width field $w_{l}(x, y)$ for which the color bars are given in $\mathrm{m}$. 

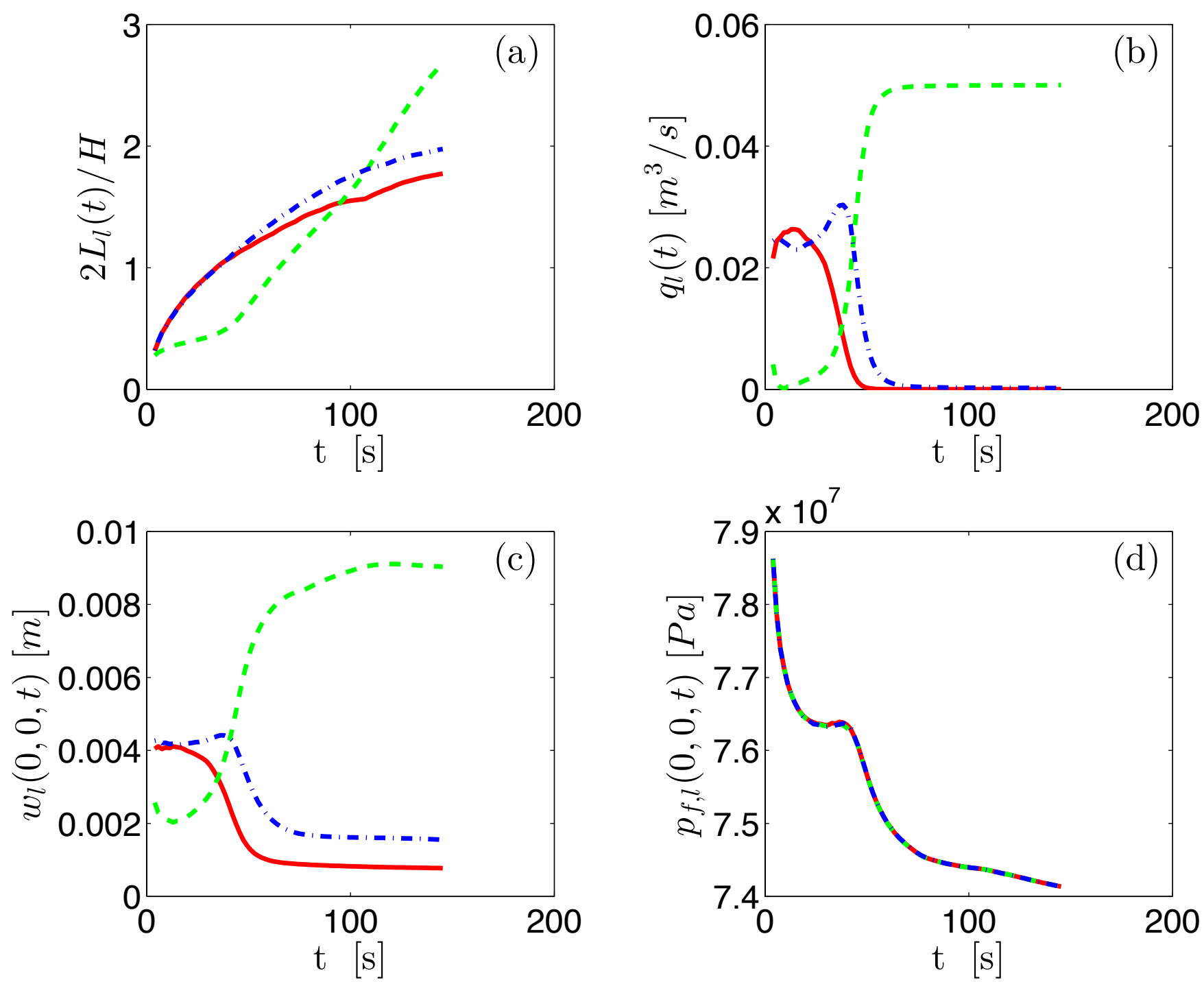

Fig. 11: Time evolution of $2 L_{l} / H, q_{l}$, and the well bore widths $w_{l}(0,0, t)$ and fluid pressures $p_{f, l}(0,0, t)$ for $N=6$ and $h_{1}=2.5, h_{2}=3.5, h_{3}=8, h_{4}=3.5, h_{5}=2.5$, $\mathrm{Red} / \mathrm{solid}$ lines are used for the fractures in planes $1 \& 6$, green/dashed lines for the fractures in planes $2 \& 5$ and blue/dash-dot lines for the fractures in planes $3 \& 4$. 


\section{Concluding remarks}

We have analyzed the simultaneous development of hydraulic fractures from multiple (zero-resistance) perforations in a single stage using a novel parallel-planar 3D model incorporating full elasto-hydrodynamic coupling and accounting for inter-fracture mechanical interactions. In particular, this model has enabled consideration of the evolution from radial to blade-like geometries with finite height-growth that is limited by symmetric stress barriers either side of a payzone. Given a prescribed total injection rate, the model is able to dynamically partition the influx of fluid into each fracture in such a way that the wellbore pressure is minimized and is the same at the inlet of each fracture in the array.

We considered the simultaneous development of fractures from arrays in which a stage has both odd $(N=5)$ and even $(N=6)$ numbers of perforations. When the perforations are uniformly distributed along the stage, the model confirms the phenomenon of stress shadowing in which the dominant fracture growth is localized in the two outer fractures, while the additional compressive stresses induced on the interior fractures stunt their growth to such an extent that they do not proceed beyond the radial propagation regime. Indeed, this localization implies that the dominant mechanism for generating new fractured area is through expanding the two book-end fractures while new area growth in the interior fractures is of little consequence due to the stress shadowing. As a result, there is little difference between the total fractured areas generated by uniform arrays having $N=3,5$, and 6 fractures.

However, we have discovered that by breaking the symmetry in the location of the perforations in the array it is possible to interfere with the localization process in a way that substantially changes the dynamics of the mutual interactions between the fractures, which helps to achieve a more uniform distribution of fractured surface across the fractures in the array. The key to achieving this is the placement of two interference fractures close to each of the two the outer fractures, which serve to constrain them from runaway localization. This strong interaction between the pairs of fractures at the edges of the array also reduces the constraint on the other interior fractures, which can be made to grow at roughly the same rate as the two book-end fractures. We find, then, that by exploiting the complex dynamics of these interacting fractures, the nonuniform array with $N=5$ is able to increase the total fractured area in the array by $46 \%$ compared to the uniform array. For the array with $N=6$ the total fractured area is increased by $74 \%$. If production turns out to scale with the fracture area, then these results show that a $\sim 50 \%-75 \%$ increase in hydrocarbon recovery is possible for the same number of perforation clusters, pumping rate, and total fluid volume when an appropriately-coupled model is employed in order to specifically chose the locations of the interference fractures, that is, the nearest-neighbors to the outer fractures in the array.

This study has highlighted the potential benefits that can be achieved by breaking the symmetry of the locations of the perforation clusters. The essential component in this analysis is the fully-coupled parallel-planar model, which is able to accurately capture the mutual mechanical interactions of the evolving hydraulic fractures and the evolution from the radial to blade-like regimes. A number of effects that have not been considered in this initial study include the stress influence of neighboring stages that have already been completed and the effect of perforation losses. These will doubtless influence the most favorable location of the interference fractures in the array, but leaves significant potential for design. It is the intention of the authors to test the modeling and design with laboratory and field experiments.

\section{References}

Abass, H.H., Soliman, M.Y., Tahini, A.M., Surjaatmadja, J., Meadows, D.L., and Sierra, L. 2009. Oriented fracturing: A new technique to hydraulically fracture an openhole horizontal well. In Proceedings SPE Annual Technical Conference and Exhibition. New Orleans, LA, USA. SPE 124483.

Baihly, J.D., Malpani, R., Edwards, C., Han, S.Y., Kok, J.C.L., Tollefsen, E.M., and Wheeler, C.W. 2010. Unlocking the shale mystery: How lateral measurements and well placement impact completions and resultant production. In Proceedings SPE Tight Gas Completions Conference. San Antonio, Texas, USA. SPE 138427.

Bunger, A.P., Jeffrey, R.G., and Zhang, X. 2011. Experimental investigation of the interaction among closely spaced hydraulic fractures. In Proceedings 45th U.S. Rock Mechanics Symposium. San Francisco, CA, USA. Paper No. 11318.

Bunger, A.P., Zhang, X., and Jeffrey, R.G. 2012. Parameters effecting the interaction among closely spaced hydraulic fractures. Soc. Pet. Eng. J. volume 17 (1): 292-306.

Bunger, A.P., Zhang, X., and Jeffrey, R.G. In Press. Constraints on simultaneous growth of hydraulic fractures from multiple perforation clusters in horizontal wells. Soc. Pet. Eng. J. .

Cipolla, C., Weng, X., Onda, H., Nadaraja, T., Ganguly, U., and Malpani, R. 2011. New algorithms and integrated workflow for tight gas and shale completions. In Proceedings SPE Annual Technology Conference and Exhibition. Denver, Colorado, USA. SPE 146872.

Crouch, S. and Starfield, A. 1983. Boundary Element Methods in Solid Mechanics. London: Unwin Hyman.

Desroches, J., Detournay, E., Lenoach, B., Papanastasiou, P., Pearson, J., Thiercelin, M., and Cheng, A.D. 1994. The crack tip region in hydraulic fracturing. Proc. Roy. Soc. London A volume 447: 39-48. 
Detournay, E. 2004. Propagation regimes of fluid-driven fractures in impermeable rocks. Int. J. Geomechanics volume 4 (1): $1-11$.

Fisher, M.K., Heinze, J.R., Harris, C.D., Davidson, B.M., Wright, C.A., and Dunn, K.P. 2004. Optimizing horizontal completion techniques in the Barnett shale using microseismic fracture mapping. In Proceedings SPE Annual Technology Conference and Exhibition. Houston, Texas, USA. SPE 90051.

Germanovich, L.N., Ring, L.M., Astakhov, D.K., Shlyopobersky, J., and Mayerhofer, M.J. 1997. Hydraulic fracture with multiple segments II: Modeling. Int. J. Rock Mech. Min. Sci. volume 34 (3-4): 472.

Kear, J., White, J., Bunger, A.P., Jeffrey, R.G., and Hessami, M.A. 2013. Three dimensional forms of closely-spaced hydraulic fractures. In A.P. Bunger, J. McLennan, and R.G. Jeffrey, editors, Effective and Sustainable Hydraulic Fracturing, chapter 34. Rijeka, Croatia: Intech.

Meyer, B. and Bazan, L. 2011. A discrete fracture network model for hydraulically induced fractures-theory, parametric and case studies. In Proceedings SPE Hydraulic Fracturing Technology Conference and Exhibition. The Woodlands, Texas, USA. SPE 140514.

Miller, C. and Waters, G. 2011. Evaluation of production log data from horizontal wells drilled in organic shales. In Proceedings SPE North American Unconventional Gas Conference and Exhibition. The Woodlands, Texas, USA. SPE 144326.

Nordgren, R. 1972. Propagation of vertical hydraulic fractures. J. Pet. Tech. volume 253: 306-314. (SPE 3009).Peirce, A. and Detournay, E. 2008. An implicit level set method for modeling hydraulically driven fractures. Computer Meth. Appl. Mech. Eng. volume 197: 2858-2885.

Peirce, A. and Detournay, E. 2008. An implicit level set method for modeling hydraulically driven fractures. Computer Meth. Appl. Mech. Eng volume 197: 2858-2885.

Perkins, T. and Kern, L. 1961. Widths of hydraulic fractures. J. Pet. Tech., Trans. AIME volume 222: 937-949.

Rice, J. 1968. Mathematical analysis in the mechanics of fracture. In H. Liebowitz, editor, Fracture, an Advanced Treatise, volume II, chapter 3, pages 191-311. New York NY: Academic Press.

Sesetty, V. and Ghassemi, A. 2013. Numerical simulation of sequential and simultaneous hydraulic fracturing. In A.P. Bunger, J. McLennan, and R.G. Jeffrey, editors, Effective and Sustainable Hydraulic Fracturing, chapter 33. Rijeka, Croatia: Intech.

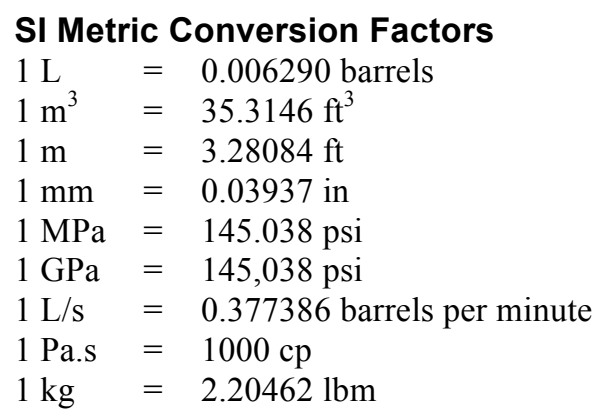

